\title{
Comportements sexuels et préventifs aux Antilles et en Guyane : un contexte peu favorable pour les femmes face au VIH/sida
}

\author{
Sandrine HALFEN ${ }^{1}$
}

\begin{abstract}
Résumé
L'analyse des données de l'enquête KABP Antilles-Guyane indique que les hommes et les femmes de ces régions ont acquis des connaissances comparables sur le $\mathrm{VIH} /$ sida. Néanmoins, les résultats relatifs aux comportements sexuels et préventifs montrent des différences importantes selon le sexe. Les femmes apparaissent particulièrement vulnérables au VIH/sida. D'une part, le multipartenariat de leur partenaire les expose indirectement au risque de contamination, et cela d'autant qu'il s'agit fréquemment d'un multipartenariat simultané et engageant des relations durables. D'autre part, les résultats relatifs à I'utilisation des préservatifs montrent que les femmes ont moins la maîtrise de la prévention que les hommes (moindre protection et utilisation des préservatifs moins étroitement liée au risque). Dans des sociétés où la différentiation sexuelle est marquée, la lutte contre le VIH/sida se doit de prendre en compte ce contexte défavorable pour les femmes et d'encourager les actions visant à initier une construction sociale et culturelle des rôles sexuels plus égalitaire.
\end{abstract}

Mots clés : Comportements sexuels, comportements préventifs, multipartenariat, vulnérabilité, inégalité.

Les données épidémiologiques relatives à l'infection à $\mathrm{VIH} /$ sida montrent que l'épidémie est particulièrement active dans les trois départements français d'Amérique (Guadeloupe, Martinique et Guyane). Le nombre moyen annuel de nouveaux cas de sida pour la période 2003-2005, rapporté à la population, y est près de sept fois plus élevé qu'en métropole : 12 cas pour 100000 habitants y ont été enregistrés contre 2 pour 100000 en métropole. De plus, de grandes disparités existent entre les trois départements, avec 31 cas pour 100000 habitants en Guyane, 8 pour 100000 en Guadeloupe et 7 pour 100000 en Martinique [1]. La transmission majoritairement hétérosexuelle du $\mathrm{VIH}$ aux Antilles et en Guyane affecte nettement les femmes puisque près de la moitié des patients vivant avec le $\mathrm{VIH} /$ sida dans départements français d'Amérique sont des femmes (48\% en moyenne, variant de $36 \%$ en Guadeloupe à $59 \%$ en Guyane) [2].

Or, les Antilles et la Guyane sont des sociétés dans lesquelles la différentiation sexuelle est particulièrement marquée, sur un

Observatoire régional de la santé d'ile-de-France, Paris. mode de domination masculine. Le contexte social apparaît très contraignant pour les femmes, où leur rôle se doit "d'évoluer dans un univers domestique et maternel » [3, p. 46]. À l'inverse, pour la population masculine, le contexte apparaît relativement permissif et « les relations multiples de l'homme semblent faire partie aux Antilles de la normalité. La plupart des femmes ne remettent pas en question ces normes et les transmettent à leurs filles " [4, p. 10]. De plus, sur le plan de l'organisation familiale, ces sociétés présentent des spécificités communes, qui trouvent principalement leurs origines dans la période esclavagiste [5], pouvant avoir un impact dans la dynamique de l'épidémie : faible nuptialité ou conjugalité [6], monoparentalité importante $[7,8]$, pluripaternité fréquente [9] etc. Enfin, les principaux indicateurs caractérisant le contexte socio-économique des Antilles et de la Guyane manifestent de fortes disparités sociales. Et les femmes de ces régions, pourtant davantage diplômées que les hommes, se trouvent plus fréquemment touchées par la précarité ${ }^{2}$. Ces situations d'insécurité sociale, conduisant fréquemment à une certaine dépendance économique vis-à-vis du conjoint, peuvent contribuer à limiter les marges de négociation dont disposent les femmes et les placer en situation de vulnérabilité, notamment face à la prévention.

Dans ce contexte, il est apparu nécessaire de déterminer les différences qui pouvaient exister entre les hommes et les femmes, sur le plan des connaissances et des comportements face au $\mathrm{VIH} / \mathrm{sida}$, afin de pouvoir mieux orienter les campagnes de prévention aux Antilles et en Guyane, en prenant davantage en compte, dans le contenu des messages, les rapports sociaux de sexe. Cette analyse est réalisée à partir des données de l'enquête Anrs-EN16-KABP départements français d'Amérique ${ }^{3}$ sur les connaissances, attitudes, croyances et comportements face au VIH/sida aux Antilles et en Guyane, conduite en 2004 par l'Observatoire régional de la santé d'île-de-France. Cette enquête a permis d'interroger par tirage aléatoire 3014 personnes dans les trois départements français d'Amérique (environ 1000 par

\footnotetext{
Emplois temporaires ou précaires, emplois à temps partiels et emplois faiblement qualifiés plus fréquents, chômage plus important, durée de chômage plus longue etc.

3 Enquête dite KABP pour Knowledge, Attitudes, Beliefs and Practices, financée par l'Anrs et la Fondation de France.
} 
département) ${ }^{4}[10]$. Les données sont comparées à celles de l'enquête KABP métropole, également conduite en 2004 par l'Observatoire régional de la santé d'Île-de-France, selon une méthodologie identique à celle utilisée pour l'enquête aux Antilles et en Guyane [11].

\section{Déclarations relatives aux comportements sexuels}

Les données de l'enquête montrent que, aux Antilles et en Guyane, les déclarations des hommes et celles des femmes présentent davantage de différences qu'en métropole. En effet, même si les connaissances des modes de transmission du VIH et la perception du risque lié au VIH/sida sont comparables entre hommes et femmes, des écarts importants peuvent être notés sur le plan des déclarations relatives aux comportements sexuels (notamment l'âge au premier rapport sexuel et le nombre de partenaires déclarés) et préventifs (notamment l'usage des préservatifs).

\section{Un début de vie sexuelle plus précoce chez les hommes que chez les femmes}

Les données relatives à l'entrée dans la sexualité, c'est-à-dire l'âge que la personne a déclaré avoir lors de son premier rapport sexuel, montrent un début de vie sexuelle plus précoce des hommes des départements français d'Amérique, comparé aux femmes de cette même région, alors qu'en métropole, garçons et filles débutent désormais à peu près au même âge leur vie sexuelle. En effet, l'âge médian au premier rapport sexuel, chez les personnes âgées de 18 à 39 ans, est, dans les départements français d'Amérique, de 15,9 ans chez les hommes et 17,4 ans chez les femmes et en métropole, respectivement de 16,6 ans et 17,2 ans. Ainsi, parmi les personnes âgées de 18 à 39 ans au moment de l'enquête, $9 \%$ des femmes avaient déjà eu un rapport sexuel à l'âge de 15 ans, par exemple, contre $30 \%$ des hommes, en Guadeloupe, respectivement $10 \%$ et $39 \%$ en Martinique, et $18 \%$ des femmes contre $44 \%$ des hommes en Guyane (figure 1). En métropole, cette proportion est d'environ $10 \%$ pour les hommes comme pour les femmes. Quand on compare les générations interrogées, les plus jeunes (18-39 ans) et les plus âgées (40-69 ans), les écarts entre les hommes et les femmes tendent néanmoins à se réduire aux Antilles et en Guyane, principalement du fait d'un abaissement de l'âge au premier rapport sexuel chez les femmes. L'âge médian au premier rapport sexuel chez les femmes est en effet passé de 18,6 ans à 17,4 ans, alors que, chez les hommes, cet âge apparaît relativement stable dans les deux générations (respectivement 16,0 ans et 15,9 ans). Cette tendance à une homogénéisation de l'âge à l'initiation sexuelle des hommes et des femmes a été observée en métropole dans les générations précédentes [12,13]. Mais, si les hommes des

\footnotetext{
Pour une présentation détaillée de la méthodologie, voir le rapport [10].
}

Antilles et de Guyane déclarent débuter plus tôt leur vie sexuelle que les femmes, leur âge au premier rapport sexuel est également plus précoce que celui des hommes de métropole, alors que les femmes des départements français d'Amérique et de métropole débutent leur vie sexuelle au même âge.

\section{Quel que soit I'âge, un multipartenariat plus fréquent chez les hommes que chez les femmes}

Les caractéristiques des comportements sexuels des hommes et des femmes diffèrent également très nettement quant aux déclarations sur le nombre de partenaires que les personnes interrogées ont indiqué avoir eus au cours de différentes périodes de leur vie (au cours des cinq dernières années, des douze derniers mois ou au moment de l'enquête).

Une des particularités majeures des comportements sexuels aux Antilles et en Guyane est la fréquence deux fois plus élevée qu'en métropole d'hommes multipartenaires, c'est-à-dire ayant déclaré avoir eu plusieurs partenaires dans l'année, que ceux-ci aient été simultanés ou successifs. Aux Antilles et en Guyane, sans différence entre les départements, $24 \%$ des hommes ont indiqué être multipartenaires contre $12 \%$ en métropole. En revanche, la proportion de femmes indiquant avoir eu plusieurs partenaires dans l'année (6\%) est comparable, dans les départements français d'Amérique et en métropole. II existe donc un écart nettement plus important, dans les départements français d'Amérique, entre les déclarations des hommes et celles des femmes. De plus, la proportion de multipartenaires tend, comme en métropole, à décroître avec l'âge chez les hommes et chez les femmes (figure 2). Néanmoins, aux Antilles et en Guyane, le multipartenariat masculin se maintient à des niveaux encore élevés aux âges avancés. A 55-69 ans par exemple, $16 \%$ des hommes dans les départements français d'Amérique ont indiqué avoir eu plusieurs partenaires dans l'année. Aussi, l'écart entre les déclarations des hommes et celles des femmes est élevé à tous les âges, et particulièrement entre 35 ans et 54 ans, alors qu'en métropole, cet écart n'est notable qu'aux classes extrêmes, c'est-à-dire parmi les personnes âgées de 18 à 24 ans et parmi celles de 55 à 69 ans.

$\mathrm{Si}$, aux Antilles et en Guyane, la fréquence déclarée du multipartenariat diffère nettement chez les hommes et chez les femmes, en revanche, les caractéristiques du multipartenariat masculin et féminin sont proches. En effet, une des spécificités du multipartenariat dans les départements français d'Amérique est qu'il implique souvent des relations simultanées et durables et des personnes vivant en couple. Parmi les personnes, hommes ou femmes, ayant eu plusieurs partenaires dans l'année, environ une sur trois a indiqué que tous ses partenaires étaient d'anciens partenaires, c'est-à-dire des partenaires avec lesquels elle avait des rapports sexuels depuis plus d'un an, alors que, chez les multipartenaires de métropole, seule une personne sur cinq 
Figure 1

Pourcentages d'hommes et de femmes âgés de 18 à 39 ans ayant déjà eu un rapport sexuel selon leur âge au moment du premier rapport
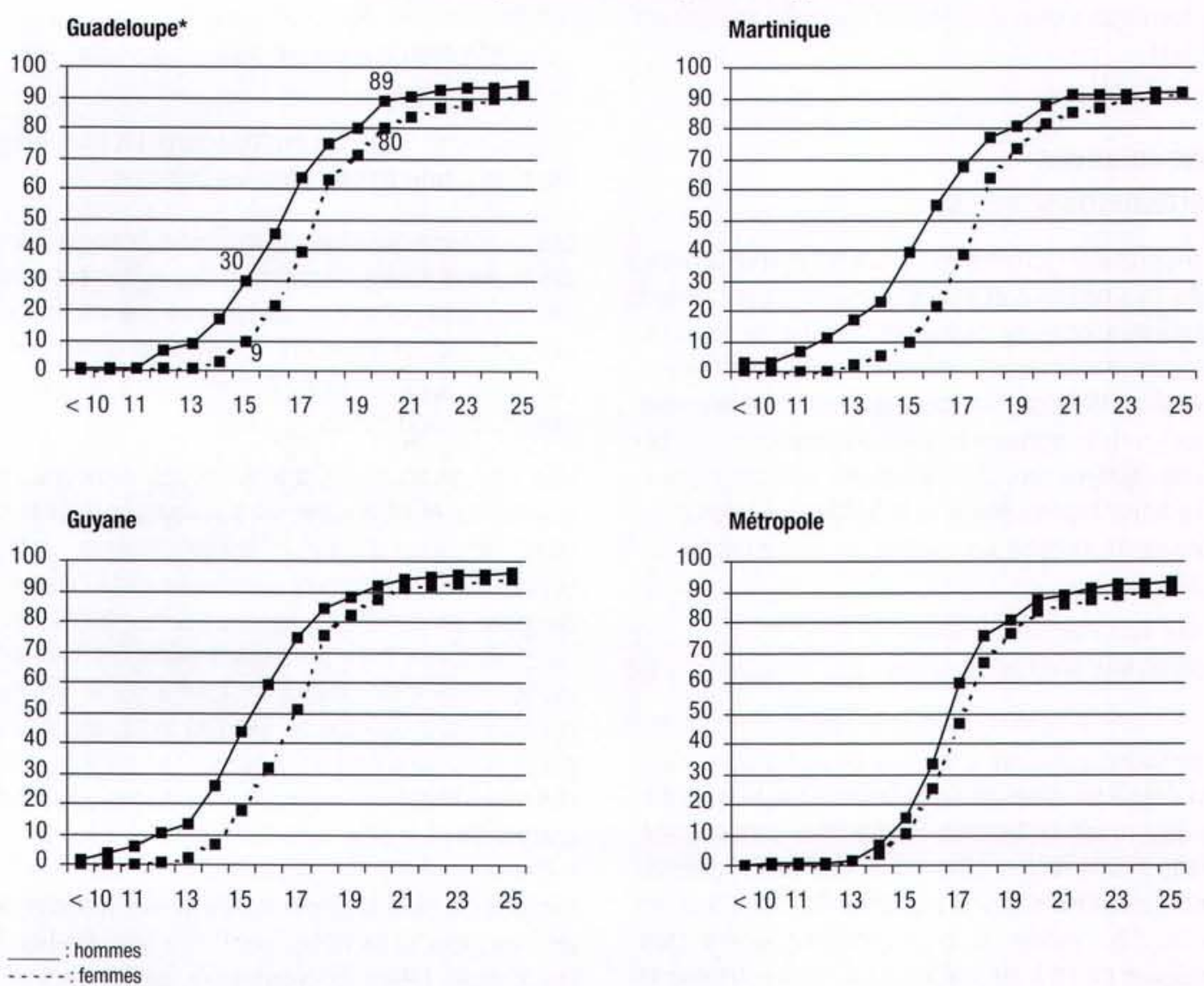

a indiqué être dans ce cas, c'est-à-dire engagée dans un multipartenariat dit stable.

Compte tenu de la fréquence du multipartenariat masculin, notamment aux âges avancés, et des caractéristiques de ce multipartenariat, $16 \%$ des hommes vivant en couple (14\% de ceux mariés et $20 \%$ de ceux non mariés) ont indiqué avoir eu plusieurs partenaires dans l'année. Ce n'est le cas que de $4 \%$ des femmes vivant en couple (respectivement $6 \%$ et $3 \%$ en métropole).

\section{Déclarations relatives à l'utilisation des préservatifs}

Des différences entre les déclarations des hommes et celles des femmes peuvent aussi être notées en ce qui concerne les comportements de prévention. Les femmes des départements français d'Amérique sont moins nombreuses que les hommes à déclarer utiliser des préservatifs, que ce soit lors de leur premier rapport sexuel ou dans des périodes récentes, alors même que leurs connaissances des modes de transmission et leur perception des risques liés au $\mathrm{VIH} /$ sida sont comparables à celles des hommes. Si certaines de ces différences peuvent être attribuées à la fréquence plus élevée du multipartenariat dans la population masculine, les données montrent que, dans des situations comparables, les femmes sont moins nombreuses que les hommes à se protéger, indiquant que d'autres éléments que les comportements sexuels différenciés entre hommes et femmes interviennent pour expliquer ces écarts.

La comparaison des données avec celles de la précédente enquête conduite aux Antilles et en Guyane en 1992 [14] montre que l'utilisation déclarée des préservatifs a nettement progressé 
Figure 2

Pourcentage de personnes ayant déclaré avoir eu au moins deux partenaires (être multipartenaires) dans les douze derniers mois

Antilles-Guyane

Hommes : $24 \%$-Femmes : $6 \%$

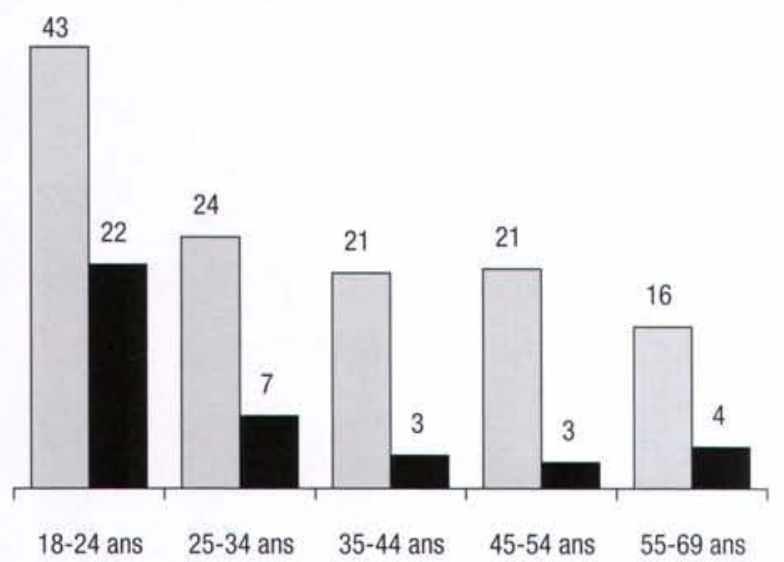

Métropole

Hommes : $12 \%$-Femmes : $6 \%$

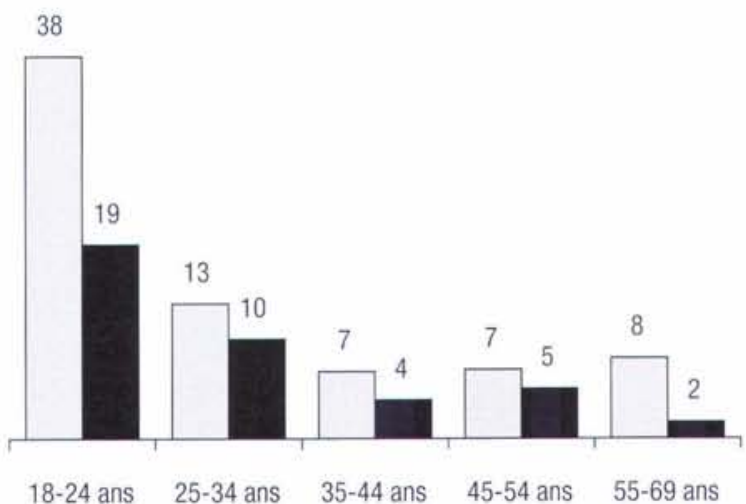

parmi la population des Antilles et de la Guyane. En 1992, 70 \% des hommes avaient indiqué avoir déjà utilisé, au moins une fois, un préservatif au cours de leur vie, ce qui est désormais le cas de $85 \%$ d'entre eux. Les femmes qui étaient moins de $50 \%$ dans ce cas en 1992 sont $72 \%$ en 2004. Les progrès, en termes de prévention, ont donc été particulièrement importants durant ces douze années parmi la population féminine et, notamment, parmi celle de Guyane. En effet, alors qu'en 1992, les femmes de Guyane étaient les moins nombreuses à avoir déjà utilisé un préservatif (39\% contre $49 \%$ des femmes de Guadeloupe comme de Martinique), elles sont, en 2004, significativement plus nombreuses que celles des Antilles à être dans ce cas (79\% contre $70 \%)$.

Dans la grande majorité des cas, l'utilisation des préservatifs, rapportée en 2004, concerne le préservatif masculin, puisque seuls $4 \%$ des hommes comme des femmes des départements français d'Amérique ont indiqué avoir déjà utilisé des préservatifs féminins et dans $67 \%$ des cas, ces personnes ont indiqué l'avoir utilisé " juste pour essayer ".

\section{Faible protection des femmes non diplômées lors de leur premier rapport sexuel}

Plus précisément, concernant l'accroissement de l'usage du préservatif, les données de l'enquête permettent de noter, chez les hommes et les femmes, une généralisation de son utilisation Iors du premier rapport sexuel. Ainsi, aux Antilles et en Guyane, comme cela est également observé en métropole, la proportion de personnes ayant utilisé un préservatif lors de leur premier rapport sexuel est d'autant plus élevée que le premier rapport sexuel s'est déroulé récemment. Dans les départements français d'Amérique, les hommes qui ont eu leur premier rapport sexuel depuis 1996 sont $80 \%$ à avoir utilisé un préservatif lors de ce rapport, alors que ce n'était le cas que de $7 \%$ de ceux ayant eu leur premier rapport sexuel avant 1985, c'est-à-dire avant la médiatisation autour du VIH/sida (figure 3). Chez les femmes, la proportion de premier rapport sexuel protégé, légèrement moindre que chez les hommes $(p=0,07)$, est de $71 \%$, alors qu'elle n'était que de $11 \%$ avant 1985 . La large diffusion de l'utilisation des préservatifs lors du premier rapport sexuel atteste de l'efficacité des actions de prévention et de communications sur le $\mathrm{VIH} /$ sida conduites au cours de ces vingt dernières années dans les départements français d'Amérique.

Néanmoins, la prévention n'a pas bénéficié de façon équivalente à tous et la protection lors du premier rapport sexuel apparaît nettement moindre parmi certaines populations des Antilles et de la Guyane, notamment parmi les femmes non diplômées. Parmi ces dernières, en effet, l'utilisation du préservatif lors du premier rapport sexuel est restée faible et a peu progressé depuis la fin des années 1980, à la différence de ce qui peut être observé parmi les femmes diplômées, qu'elles aient ou non le baccalauréat (figure 4). Ainsi, parmi les femmes qui ont eu leur premier rapport sexuel depuis 1996, seules $40 \%$ de celles non diplômées ont utilisé un préservatif lors de ce premier rapport 
Figure 3

Pourcentage de personnes ayant déclaré avoir utilisé un préservatif

lors de leur premier rapport sexuel

selon la date de ce premier rapport (en \%)

Antilles-Guyane

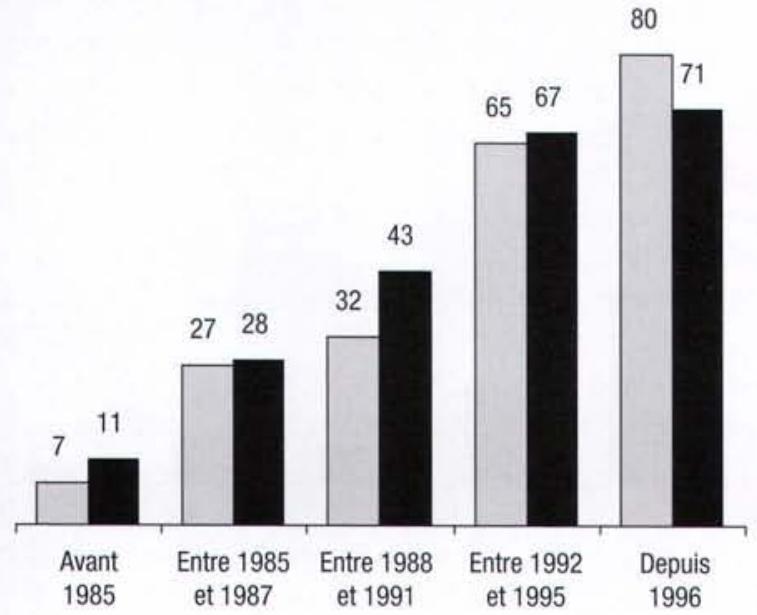

Métropole

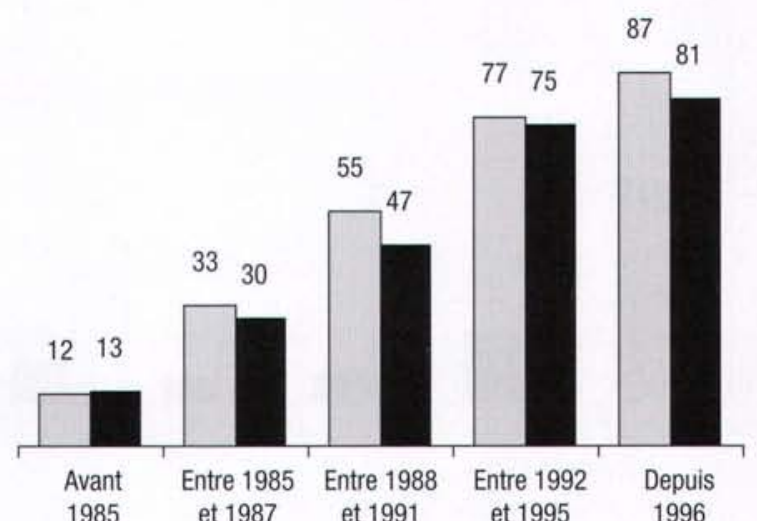

Hommes

Femmes

Figure 4

Pourcentage de personnes aux Antilles et en Guyane ayant déclaré avoir utilisé un préservatif lors de leur premier rapport sexuel

selon la date de ce premier rapport et leur diplôme (en \%)

Hommes

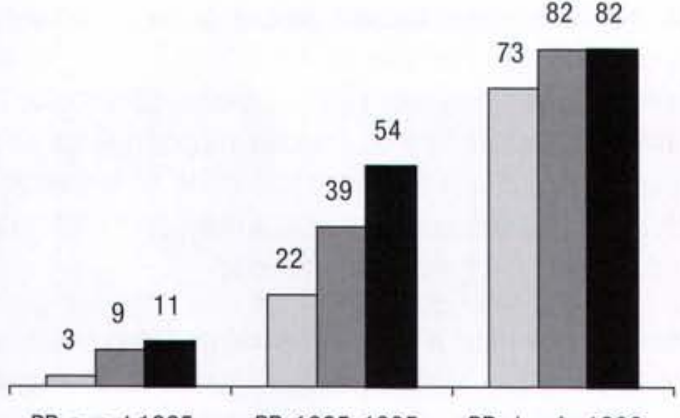

PR avant 1985 PR 1985-1995 PR depuis 1996
Femmes

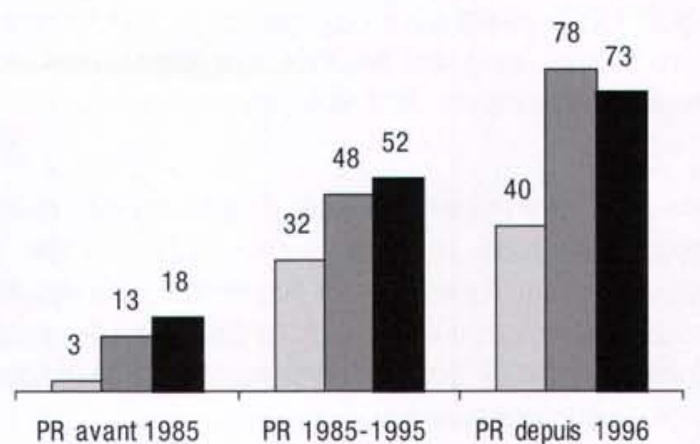

Sans diplôme

Diplôme inférieur au baccalauréat

Baccalauréat ou plus 
sexuel, alors que cette proportion est proche du double parmi les femmes ayant au moins un diplôme $(74 \%, p=0,004)$. Dans la population masculine, en revanche, la progression de l'utilisation du préservatif lors du premier rapport sexuel a bénéficié à tous les hommes, que ceux-ci soient ou non diplômés.

Or, si l'utilisation des préservatifs lors du premier rapport sexuel constitue un indicateur permettant de mesurer la prise en compte du risque du VIH lors de l'initiation sexuelle, elle constitue aussi, et surtout, un facteur prédictif fort d'une utilisation ultérieure des préservatifs $[15,10$ p. 169].

\section{Moindre protection des femmes multipartenaires}

L'analyse de l'utilisation récente des préservatifs montre que les personnes qui ont eu plusieurs partenaires durant les douze mois précédant l'enquête, les multipartenaires, sont nettement plus nombreuses que celles qui ont eu un seul partenaire à déclarer avoir utilisé des préservatifs, au moins une fois durant cette période. Cette tendance, qui manifeste une certaine prise en compte du risque du $\mathrm{VIH}$, se retrouve chez les hommes comme chez les femmes, dans des proportions comparables à ce qui est observé en métropole, et sans différence entre les trois départements. Aux Antilles et en Guyane, ce sont donc $25 \%$ des monopartenaires qui ont déclaré avoir utilisé au moins une fois des préservatifs dans l'année (27\% des hommes et $23 \%$ des femmes, écart non significatif) contre $73 \%$ des multipartenaires ( $75 \%$ des hommes et $64 \%$ des femmes, écart non significatif).

Chez les seul(e)s multipartenaires, des niveaux différents d'utilisation des préservatifs peuvent être notés, selon les caractéristiques des individus. Ces différences permettent d'observer, d'une part, que certaines catégories de multipartenaires, que ce soit chez les hommes ou chez les femmes, se protègent moins que les autres face au VIH/sida, et, d'autre part, que chez les multipartenaires, à caractéristiques comparables, les femmes sont toujours moins nombreuses que les hommes à déclarer utiliser des préservatifs.

En ce qui concerne le premier aspect, chez les hommes comme chez les femmes, un déficit de protection est observé, en particulier parmi les multipartenaires les plus âgés (45-69 ans) et parmi les multipartenaires les moins diplômés. Ainsi, par exemple, si en moyenne $64 \%$ des femmes multipartenaires ont utilisé au moins une fois des préservatifs dans l'année, cette proportion varie de $34 \%$ parmi les femmes multipartenaires sans diplôme à $59 \%$ chez celles ayant un diplôme inférieur au baccalauréat, puis $76 \%$ chez celles ayant au moins le baccalauréat. Chez les hommes multipartenaires, qui sont en moyenne $75 \%$ à avoir utilisé des préservatifs dans l'année, les proportions sont respectivement de $53 \%, 71 \%$ et $90 \%$. On peut aussi noter que, parmi les femmes multipartenaires qui ne vivent pas en couple, celles en situation de monoparentalité semblent être moins nombreuses à avoir utilisé des préservatifs dans l'année que celles qui ne sont pas dans cette situation ( $64 \%$ contre $84 \%, p=0,07$ ) et cette tendance peut s'observer à diplôme comparable.

En ce qui concerne la moindre protection des femmes et, donc, leur plus grande exposition au risque comparée aux hommes, si on se réfère, par exemple, à l'âge, on note que parmi les multipartenaires, les femmes de 45 à 69 ans ne sont que $17 \%$ à avoir utilisé des préservatifs dans l'année, alors que cette proportion est de $54 \%$ chez les hommes de cette même classe d'âges $(p=0,003)$. Le modèle de régression final cherchant à identifier, chez les multipartenaires, les déterminants de l'utilisation des préservatifs (tableau 1) montre nettement que, à caractéristiques comparables (âge, diplôme, origine géographique, situation conjugale, importance accordée à la religion ou nombre de nouveaux partenaires), les hommes multipartenaires ont une probabilité nettement supérieure à celle des femmes (odd ratio ajusté : 3,9 ; intervalle de confiance (IC) à $95 \%: 1,9$ $7,7)$ d'avoir utilisé des préservatifs au cours des douze mois précédant l'enquête. Le modèle de régression atteste également d'une moindre protection parmi les multipartenaires les plus âgés, comparés aux plus jeunes (ORa : 0,2 ; IC $95 \%$ : 0,1-0,5) et, à l'inverse, d'une bien meilleure protection des multipartenaires les plus diplômés, comparés à ceux sans diplôme (ORa : 6,5 ; IC $95 \%: 2,3-18,6)$. Cette même tendance peut être retrouvée

\section{Tableau 1}

Modèle final d'une régression logistique effectuée parmi les multipartenaires des Antilles et de Guyane où la variable dépendante est le fait d'avoir utilisé, au moins une fois, des préservatifs au cours des douze mois précédant l'enquête

\begin{tabular}{lll}
\hline \multicolumn{1}{c}{ Multipartenaires Antilles-Guyane $(\mathbf{n = 4 3 3 )}$} & OR ajusté & IC à $95 \%$ \\
\hline Femmes & 1 & \\
Hommes & $3,9^{* * *}$ & {$[1,9-7,7]$} \\
$18-34$ ans & 1 & \\
$35-44$ ans & 0,7 & {$[0,3-1,6]$} \\
$45-69$ ans & $0,2^{* * *}$ & {$[0,1-0,5]$} \\
Sans diplôme & 1 & \\
Diplôme inférieur au baccalauréat & 1,9 & {$[0,8-4,6]$} \\
Baccalauréat & $5,1^{* *}$ & {$[1,7-15,8]$} \\
Etudes supérieures & $6,5^{* * *}$ & {$[2,3-18,6]$} \\
Nés hors des DFA dans la Caraỉbe / & 1 & \\
$\quad$ Amérique du Sud & 0,8 & {$[0,2-2,6]$} \\
Nés dans les DFA & 0,3 & {$[0,1-1,6]$} \\
Nés en métropole & 0,5 & {$[0,7-2,9]$} \\
Autres & 1 & \\
Mariés cohabitants & 0,9 & {$[0,3-2,1]$} \\
Non mariés cohabitants & 1,7 & {$[0,8-3,9]$} \\
Non cohabitants & 1 & \\
Religion très importante & 1,8 & {$[0,7-4,7]$} \\
Importante & $4,5^{*}$ & {$[1,4-14,2]$} \\
Pas importante & 1 & \\
Aucun nouveau partenaire & $2,7^{* *}$ & {$[1,4-5,2]$} \\
Au moins un nouveau partenaire &
\end{tabular}

$\cdot p<0,05 \quad \cdots p<0,01 \quad \cdots p<0,001$.

DFA : départements français d'Amérique ; IC : indice de confiance. 
chez les hommes comme chez les femmes, dans des modèles de régressions réalisés séparément (résultats non présentés).

\section{Chez les femmes, une utilisation des préservatifs avec leurs nouveaux partenaires peu corrélée au risque}

Les données relatives à l'utilisation de préservatifs lors du dernier rapport sexuel apportent des précisions sur les facteurs qui peuvent favoriser ou, au contraire, freiner l'utilisation des préservatifs, dans un contexte et avec un partenaire particuliers.

Les analyses permettent de noter que l'utilisation déclarée des préservatifs chez les femmes semble moins étroitement liée au risque (perçu ou réel) de contamination par le VIH que ce qui est observé chez les hommes. En raisonnant, par exemple, uniquement parmi les personnes ayant décrit un dernier partenaire du sexe opposé, connu depuis moins d'un an, I'utilisation déclarée des préservatifs lors du dernier rapport sexuel, chez les hommes, semble d'autant plus importante que ceux-ci perçoivent une situation de risque (méconnaissance des antécédents de dépistage de leur partenaire, perception du multipartenariat de leur partenaire). Chez les femmes en revanche, la protection ne semble pas varier selon le risque, perçu ou réel. La proportion de femmes ayant indiqué avoir utilisé un préservatif lors du dernier rapport sexuel n'est pas supérieure chez celles qui pensent que leur partenaire est multipartenaire, comparé à celles qui sont sûres que leur partenaire n'a pas d'autres partenaires. De même, les femmes qui savent que leur partenaire n'a pas fait de test de dépistage du VIH ou celles qui ignorent s'il en a fait un n'ont pas été plus nombreuses à se protéger (respectivement $53 \%$ et $47 \%$ ) que celles qui savent que leur partenaire a fait un test de dépistage ( $46 \%$ ). Chez les hommes, les proportions sont respectivement de $64 \%$ et $79 \%$ versus $43 \%(p=0,0004)$ indiquant une meilleure protection parmi les hommes méconnaissant les antécédents de dépistage de leur partenaire. De plus, la protection des femmes est significativement moindre quand il y a eu une consommation d'alcool juste avant le rapport sexuel, y compris quand cette consommation ne concernait que le partenaire. Ainsi les femmes qui ont déclaré que leur partenaire avait consommé de l'alcool juste avant le rapport sexuel ont été $21 \%$ à utiliser un préservatif lors de ce rapport contre $55 \%$ de celles qui ont indiqué que leur partenaire n'avait pas consommé d'alcool ( $p=0,0003)$. Chez les hommes, en revanche, la protection est comparable chez ceux qui indiquent que leur partenaire avait consommé de l'alcool juste avant le rapport sexuel et chez ceux qui indiquent que leur partenaire n'avait pas consommé d'alcool (respectivement $65 \%$ et $63 \%$ ).

\section{Conclusion}

Les analyses produites à partir des données de l'enquête permettent de noter que, globalement, aux Antilles et en Guyane, les hommes et les femmes ont acquis des connaissances comparables sur le $\mathrm{VIH} /$ sida, tant sur les modes de transmission du virus que sur l'efficacité des moyens à mettre en œuvre pour lutter contre l'infection ou encore sur la connaissance de l'existence des traitements.

Néanmoins, les résultats relatifs aux comportements, sexuels ou préventifs, témoignent de différences importantes entre les hommes et les femmes des Antilles et de Guyane. Ainsi dans les départements français d'Amérique, l'âge au premier rapport sexuel des hommes reste plus précoce que celui des femmes, même si la tendance semble aller, comme c'est déjà le cas en métropole, vers une homogénéisation des comportements. D'un écart de deux à trois ans en moyenne entre les hommes et les femmes des départements français d'Amérique âgés de 40 ans et plus, celui-ci est désormais d'un an environ pour les générations de moins de 40 ans. Mais, c'est surtout sur la déclaration du nombre de partenaires que les différences entre les hommes et les femmes sont particulièrement marquées. En effet, la proportion de personnes ayant indiqué avoir eu plusieurs partenaires au cours des douze mois précédant l'enquête est quatre fois moindre chez les femmes que chez les hommes $6 \%$ contre $24 \%$ ), voire même sept fois moindre parmi les personnes âgées de 35 à 54 ans (3 \% contre $21 \%$ ). Si le multipartenariat est plus fréquent parmi les personnes ne vivant pas en couple, la persistance du multipartenariat masculin aux âges avancés conduit à ce qu'une proportion relativement élevée d'hommes vivant en couple (un sur six) ait déclaré avoir eu plusieurs partenaires dans l'année. Comme pour les autres questions de l'enquête, il s'agit de données déclaratives, mais c'est probablement sur le nombre déclaré des partenaires que les contextes normatifs peuvent jouer différemment chez les hommes et chez les femmes. II est généralement admis, pour interpréter ces écarts communs à la plupart des enquêtes portant sur la sexualité $[16,17]$, que les individus, hommes et femmes, comptabiliseraient leurs partenaires différemment : les hommes tendraient à comptabiliser l'ensemble des partenaires avec lesquelles ils ont eu au moins un rapport sexuel (voire à sur-estimer quelque peu leur nombre) et les femmes tendraient à ne comptabiliser que les partenaires qui ont " compté » pour elles (voire à sous-estimer quelque peu leur nombre). L'importance du décalage entre les déclarations des hommes et celles des femmes aux Antilles et en Guyane révèlent très probablement le contexte normatif, beaucoup plus contraignant pour les femmes qu'en métropole où le multipartenariat féminin, assimilé à de l'infidélité, est largement stigmatisé. La femme multipartenaire est alors perçue " comme la femme infidèle non seulement à l'homme, mais également infidèle à l'ordre naturel, à l'ordre religieux, à l'ordre spatial, à l'ordre moral et à l'ordre social » [3, p. 239]. À l'inverse, le multipartenariat masculin apparaît relativement valorisé et comme le souligne J André au sujet de la " famille noire antillaise ", cité par M Giraud [18] «... hommes et femmes sont soumis à deux régimes de leur vie sexuelle nettement distincts. Aux uns la pluralité, aux autres la fidélité. La femme mariée, la concubine (...) n'ont pas 
de liaison extra-résidentielle, sauf à courir le risque de la honte et de l'opprobre - là où, à l'inverse, l'homme construit l'essentiel de sa réputation. ".

L'analyse des données de l'enquête Vespa-départements français d'Amérique relatives aux personnes séropositives prises en charge dans les services hospitaliers des Antilles et de Guyane montre que les différences hommes/femmes, observées dans la population générale, en termes de comportements sexuels, tendent à se maintenir parmi les personnes séropositives, cela malgré l'impact majeur que l'infection a pu entraîner, en particulier sur leur vie conjugale et sexuelle [19]. Parmi les personnes séropositives interrogées dans les départements français d'Amérique et s'étant déclarées hétérosexuelles, les hommes sont près de trois fois plus nombreux que les femmes à déclarer avoir eu plus de cinq partenaires au cours de leur vie (67\% contre $24 \%$ ). Et si on se réfère à l'année précédant l'enquête, la proportion de multipartenaires est de $21 \%$ chez les hommes contre $8 \%$ chez les femmes $(p=0,0009)$, soit des écarts relativement proches de ceux notés en population générale [20].

Dans le contexte de l'épidémie de VIH/sida, les données de l'enquête KABP Antilles-Guyane montrent une vulnérabilité importante des femmes de ces régions face à l'infection. D'une part, le multipartenariat de leur partenaire les expose indirectement au risque de contamination, et cela d'autant qu'il s'agit fréquemment d'un multipartenariat simultané et engageant des relations durables. Or, les préservatifs sont d'autant moins utilisés que les relations sont anciennes et, donc, probablement jugées plus sûres.

D'autre part, les résultats relatifs à l'utilisation des préservatifs montrent que, aux Antilles et en Guyane, les femmes ont moins la maîtrise de la prévention que les hommes : moindre protection lors du premier rapport sexuel, moindre protection chez les femmes multipartenaires, utilisation des préservatifs moins étroitement liée au risque que chez les hommes. Ces résultats semblent indiquer que, dans les départements français d'Amérique, le fait d'utiliser un moyen de protection comme le préservatif (d'autant plus lorsqu'il s'agit d'un préservatif masculin) dépend le plus souvent du choix des hommes et que ce choix est principalement raisonné en fonction de la perception que les hommes ont de la situation. Parmi les multipartenaires, les femmes ont d'ailleurs été deux fois plus nombreuses que les hommes à déclarer avoir été confrontées à un refus d'utilisation des préservatifs de la part d'un de leurs partenaires au cours des cinq dernières années ( $33 \%$ contre $17 \%, p=0,006$ ). Cela montre la difficulté qu'il peut y avoir, pour les femmes, dans la négociation autour de l'utilisation des préservatifs et illustre l'interrogation de Maryse Condé " Comment la femme antillaise, plus qu'une autre entravée par son éducation mutilante, pourra-t-elle s'affirmer en face du mâle ? " [21, p. 28]. Cette marge de négociation semble être d'autant plus étroite que les femmes se trouvent en situation de vulnérabilité sociale, et donc potentiellement dans une relation de dépendance économique (et/ou affective) à leur conjoint ou leur partenaire. C'est sans doute ce qui peut expliquer la faible protection des femmes non diplômées lors de leur premier rapport sexuel (si on la compare par exemple à celle des hommes non diplômés), la faible utilisation des préservatifs chez les femmes multipartenaires sans diplôme ou encore chez celles en situation de monoparentalité. Chez les femmes en situation de dépendance, c'est probablement la crainte d'une mise à l'écart par le partenaire qui peut limiter les possibilités de négociation autour du préservatif. Et parmi les femmes séropositives, c'est probablement cette même crainte qui peut conduire certaines d'entre elles à taire leur séropositivité. Dans l'enquête Vespadépartements français d'Amérique, parmi les personnes vivant en couple, les femmes sont en effet plus nombreuses que les hommes à ne pas avoir révélé leur séropositivité à leur partenaire (20\% contre $12 \%, p=0,02)$ [19].

La précarité des femmes accroît donc leur vulnérabilité face à l'épidémie, dans un contexte de domination masculine très marquée. Et c'est probablement sur le terrain de la sexualité que cette domination trouve à s'exprimer et peut s'exercer de façon privilégiée: premiers rapports sexuels forcés (deux fois plus fréquemment cités par les femmes des départements français d'Amérique que par celles de métropole), exposition au risque de contamination du fait du multipartenariat des conjoints, refus de protection, isolement des femmes séropositives au sein de leur couple.

La lutte contre le VIH/sida aux Antilles et en Guyane se doit de prendre en compte ce contexte défavorable pour les femmes et d'encourager les actions visant à initier une construction sociale et culturelle des rôles sexuels plus égalitaire.

\section{Références bibliographiques}

1. InVS. Déclaration des cas de sida au 30 juin 2006 , Insee, population estimée au

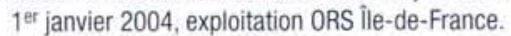

2. Bouillon K, Lert F, Michelot F, Schmaus A, Spire B, Dray-Spira R. Les patients vivant avec le VIH-sida dans les départements français d'Amérique : résultats de l'enquête Anrs-Vespa, 2003. BEH novembre 2005 ; 46-47 : 240-242.

3. Bombereau G. Représentations sociales $d u \mathrm{VIH} /$ sida en Guadeloupe et recommandations à l'usage de la santé publique. La peur ou la mort dans l'âme dans les Antilles françaises, Thèse de doctorat es sciences de l'éducation, Université Laval, Québec, Université René Descartes, Paris V, 2005, 384 p.

4. Alibar F, Lembeye-Boy P. Le couteau seul... Sé Kouto sèl..., La condition fẻminine aux Antilles, Volume II : Vies de femmes. Paris : Editions caribéennes, $1982,280 \mathrm{p}$.

5. Charbit Y. Famille et nuptialité dans la Caraibe. Paris: INED / PUF, Travaux et documents, $n^{\circ} 114,1987,414 \mathrm{p}$.

6. Gautier A. Procreation et famille en Guadeloupe et dans la Caraibe. Espace Populations Sociétés, Regards vers l'outre-mer français $2004 ; 4$; 207-219.

7. Génix D. Femmes d'aujourd'hui. Antiane, Insee Antilles-Guyane, $n^{\circ} 60$, juin 2004, 15-17. 
8. Guillaume M. La monoparentalité et ses difficultés au féminin. Antiane, Insee Antilles-Guyane, $n^{\circ} 52$, mai 2002, 7-10.

9. Diman-Antenor D, Jannot MF. Lorsque l'enfant paraît... Antiane, Insee AntillesGuyane, $n^{\circ} 30$, décembre 1995, p. 19-22.

10. Halfen $\mathrm{S}$, Fénies K, Ung B, Grémy I. Les connaissances, attitudes, croyances et comportements face au V/H/sida aux Antilles et en Guyane en 2004. Rapport ORS île-de-France, avril 2006, $290 \mathrm{p}$.

11. Beltzer N, Lagarde $M$, Wu-Zhou $X$, Grémy I. Les connaissances, attitudes, croyances et comportements face au VIH/sida en France en 2004, évolutions 1992 - 1994 - 1998 - 2001 - 2004, rapport ORS lle-de-France, novembre $2005,178 p$

12. Bozon M. A quel âge, les femmes et les hommes commencent-ils leur vie sexuelle? Comparaisons mondiales et évolutions récentes. Population et Sociétés $2003 ; 391: 1-4$.

13. Bajos $N$, Bozon $M$, Beltzer $N$ et l'équipe CSF. Contexte de la sexualité en France. Dossier de presse : premiers résultats de l'enquête CSF. Paris, Anrs, Inserm, Ined, 13 mars 2007 (www.inserm.fr).

14. Giraud $\mathrm{M}$, Gilloire $\mathrm{A}$, Halfen $\mathrm{S}$, de Colomby $\mathrm{P}$. Analyse des comportements sexuels aux Antilles et en Guyane (ACSAG). Rapport Anrs, document multicopié, octobre 1994, $107 \mathrm{p}$.
15. Grémy I, Beltzer N. HIV risk and condom use in the adult heterosexual population in France between 1992 and 2001 : return to the starting point ? ", AIDS $2004 ; 18: 805-809$.

16. Leridon $\mathrm{H}$. Nombre, sexe et type de partenaires. In Les comportements sexuels en France, A Spira, N Bajos et le Groupe ACSF, Eds. Paris : La documentation Française, 1993, p. 133-141.

17. Levinson S. L'organisation temporelle des premières relations sexuelles. In L'entrée dans la sexualité. Le comportement des jeunes dans le contexte du sida, H Lagrange, B Lhomond, Eds. Paris : La Découverte, 1997, p. 227-254.

18. Giraud M. Une construction coloniate de la sexualité. À propos du multipartenariat hétérosexuel caribéen. Actes de la recherche en sciences sociales $1999 ; 128: 46-55$

19. Masse V, Dray-Spira R, Spire B, Schmaus A, Lert F. Vie de couple, vie sexuelle et prévention chez les hétérosexuels vivant avec le VIH-sida aux Antilles et en Guyane, Résultats de l'enquête Anrs-Vespa, 2003. BEH 2005 ; 46-47 : 243244

20. Lert F, Sitta R. Enquête Anrs-Vespa 2003 aux Antilles et en Guyane. Communication personnelle, mars 2007

21. Condé M. La parole des femmes, Essai sur des romancières des Antilles de langue française. Paris : L'Harmattan, 1993, p. 132. 